\title{
Hall resistance anomalies in the integer and fractional quantum Hall regime
}

\author{
E. Peraticos $\odot,{ }^{*}$ S. Kumar $\odot$, and M. Pepper \\ Department of Electronic and Electrical Engineering, University College London, Torrington Place, \\ London WC1E 7JE, United Kingdom \\ and London Centre for Nanotechnology, 17-19 Gordon Street, London WC1H OAH, United Kingdom
}

A. Siddiki

Department of Electrical and Electronics Engineering, Maltepe Üniversitesi, Marmara Egitim Köyü, Maltepe, Istanbul 34857, Turkey

I. Farrer, ${ }^{\dagger}$ D. Ritchie, G. Jones, and J. Griffiths

Cavendish Laboratory, JJ Thomson Avenue, Cambridge CB3 OHE, United Kingdom

(Received 11 June 2020; revised 19 August 2020; accepted 3 September 2020; published 23 September 2020)

\begin{abstract}
Experimental evidence of resistance anomalies in the high-mobility two-dimensional electron gas (2DEG) formed in the GaAs/AlGaAs heterostructure, in the integer and fractional quantized Hall regime, is shown. The data complement to a good approximation the semianalytic calculations used to describe the formation of integral and fractional incompressible strips. The widths of current-carrying channels were calculated by incorporating the screening properties of the 2DEG and the effect of a magnetic field in the perpendicular mode. The manybody effects of the composite fermions are taken into consideration for the energy gap for the fractional states. It is shown that incompressible strips at the edges for both integer and fractional filling factors coexist in their evanescent phase for a particular range of magnetic fields, resulting in overshoot effects at the Hall resistance. Specifically, anomalous Hall resistances were noticed for filling factors $v=\frac{4}{3}, \frac{3}{2}, \frac{5}{3}, \frac{8}{3}, 3, \frac{10}{3}, \frac{7}{2}$, and 5 . This effect is explained and discussed using the screening theory.
\end{abstract}

DOI: 10.1103/PhysRevB.102.115306

\section{INTRODUCTION}

Since the discovery of the integer/fractional quantum Hall effect (IQHE/FQHE) [1,2], the study of quantum states in the two-dimensional electron gas (2DEG) systems has sparked great interest, particularly utilizing its quantum-mechanical properties for applications in quantum computing [3]. However, a universal theory has still not materialized to explain the various anomalous phenomena that appear in experimental studies such as the anomalous Hall resistance (overshooting) [4-6]. The resistance overshooting is the observance of an anomalous increase in resistance at a plateau corresponding to a specific filling factor $v$ observed within the transverse resistance of the IQHE/FQHE. The importance of studying this phenomenon is that it can clarify our understanding of the formation of quantum states within these regimes. Over the years there has been development of a theoretical explanation of this phenomenon, with data eventually showing great similarities between experimental and theoretical work for IQHE. However, for FQHE, although theories predicted overshooting in the fractional states, the phenomenon has not been observed or studied extensively. In this paper we show the observation of overshooting or anomalous Hall resistance

\footnotetext{
*elias.peraticos.10@ucl.ac.uk

${ }^{\dagger}$ Also at Department of Electronic and Electrical Engineering, The University of Sheffield, Sheffield S10 2TN, United Kingdom.
}

in the FQHE regime and present a theoretical model based on compressible/incompressible states. These states are areas of different characteristics which appear within the system as a consequence of the screening effect, with properties similar to those of conducting and insulating regions. Within this framework electron interaction effects, specifically Coulomb interactions, are included, providing a close match between theoretical and experimental work.

This paper has been arranged in the following manner: In Sec. II the sample's structure and geometry and the experimental setup and methods are presented. Section III covers the explanation of the anomalous resistance overshoot and the various theories surrounding it. In Sec. IV, a discussion comparing theory and experimental results from other groups with data collected from our sample is given. Section V concludes our findings.

\section{SAMPLE AND METHODS}

In the present work we have utilized the $\mathrm{GaAs} / \mathrm{Al}_{0.33} \mathrm{Ga}_{0.67} \mathrm{As}$ heterostructure grown by molecularbeam epitaxy. A 2DEG is formed at the interface of GaAs and AlGaAs at a depth of around $100 \mathrm{~nm}$ from the surface of the heterostructure. The electron carrier density was calculated in the dark (light) to be $n_{0}=1.6 \times 10^{11} \mathrm{~cm}^{-2}\left(4.45 \times 10^{11}\right.$ $\left.\mathrm{cm}^{-2}\right)$, and its mobility $\mu_{e}=0.37 \times 10^{6} \mathrm{~cm}^{2} / \mathrm{V}\left(1.05 \times 10^{6}\right.$ $\mathrm{cm}^{2} / \mathrm{V} \mathrm{s}$ ). A red light-emitting diode was used to illuminate the experimental device. The heterostructure was converted 


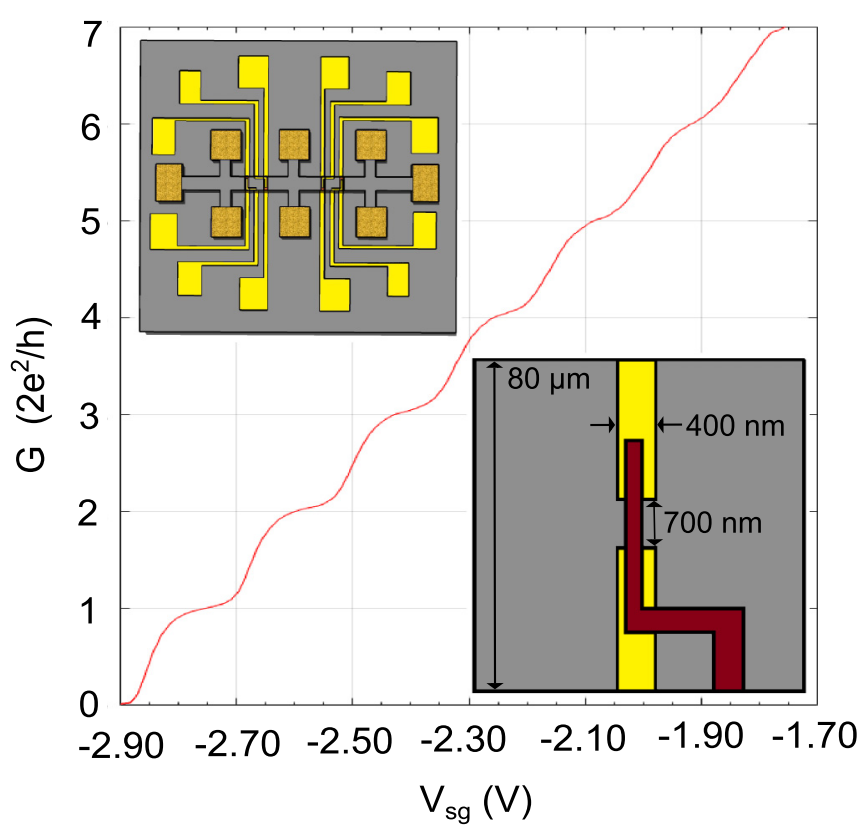

FIG. 1. Plot of conductance $G$ in units of $2 e^{2} / \mathrm{h}$ versus $V_{s g}$, with the signature quantized conductance plateaus for a $1 \mathrm{D}$ system. The insets are schematics of the device used in this paper. The upper inset shows the Hall bar. The dark yellow rectangles are the Ohmic contacts, and the bright yellow structures are the titanium/gold optical gates. These are used to create contact with the split gates and top gates, seen in a zoomed-in picture in the lower inset. The width of the Hall bar is $W_{H}=80 \mu \mathrm{m}$, and the length is $L_{H}=1400 \mu \mathrm{m}$. The split gates (shown in yellow) are $700 \mathrm{~nm}$ apart and have a width of $400 \mathrm{~nm}$. The top gate (dark brown) is separated from the split gates via PMMA.

into a Hall bar, as shown in Fig. 1. A pair of split gates was fabricated through the standard lithographic methods (photo- and electron-beam lithography) [7,8], insulated by poly(methyl methacrylate) (PMMA) from the top gate. The width of the top gate is the same as the split gates (drawn smaller in Fig. 1 in order to make the design clearer). The distance between the split gate and the $R_{x y}$ contacts is 300 $\mu \mathrm{m}$. The distance between the $R_{x x}$ contacts is $1080 \mu \mathrm{m}$; the distance between the split gates and the left $R_{x x}$ Ohmic is $230 \mu \mathrm{m}$, and the distance to the right Ohmic is 850 $\mu \mathrm{m}$. The split gates and the top gate are used to create a quasi-one-dimensional (1D) channel using the 2DEG $[7,8]$. The magnetotransport measurements were performed in a dilution refrigerator at a base temperature of $10 \mathrm{mK}$, unless stated otherwise.

The conductance as a function of voltage applied on the split gates $V_{s g}$ is shown in Fig. 1. This was used as the reference for $V_{s g}$ applied in the measurements described in the following sections. The longitudinal, $R_{x x}$, and transverse, $R_{x y}$, resistances of the sample were measured using the standard four-terminal method.

\section{HALL RESISTANCE ANOMALY (OVERSHOOT)}

The study of QH effect is of vital importance due to its importance in metrology as a standard of resistance. Nonetheless, although the IQHE is explained satisfactorily, to some extent, by the 1D Landauer-Büttiker formalism $[9,10]$ of edge states, it is limited in describing various experimental phenomena that contradict it. One such phenomenon is the resistance overshoot in the IQHE which has been extensively studied, both experimentally and theoretically. Resistance overshooting is the observation of nonmonotonic increase of Hall resistance at the lower end of the magnetic field of the quantized plateaus, forming at integer filling factors which are defined by the number of occupied quantized (spinresolved) Landau levels (LLs) below the Fermi energy. Over the years various explanations for this phenomenon have been provided but have failed to provide strong evidence for their validity. Some examples are the suggestion of nonideal probes [10-12], the decoupling between the magnetic fields of the two edge states associated with the topmost spin-split LL [13], and the nonequilibrium population of electrons at sample boundaries due to bulk properties causing scattering between edge states together with spin-orbit interactions [14].

In contrast, the screening theory which takes into account the Coulomb interactions between charged carriers seems to illustrate this phenomenon within a framework which follows closely the experimental observations and how it changes with strong magnetic fields [15-19]. This was initially proposed in a qualitative manner by Beenakker [20] and Chang [21] considering the electron gas being divided into strips which alternate between incompressible (IS) and compressible (CS) states. However, Beenakker stated then that this would not be the case for fractional states [20]. In strong contrast, Chklovskii et al. [22] argued that these states can successfully explain the IQHE. Today, however, it is understood that a similar model can explain these states as well by taking into consideration the composite fermion (CF) model.

The CS and IS are formed at magnetic fields for a spatially constrained 2DEG, resulting in the Fermi energy alternating between a position that overlaps with the LL and regions where it lies between the consecutive energy levels, respectively. The latter IS is comparable to an insulating region which is formed at the plateaus of the quantum Hall resistance corresponding to specific filling factor $v$ and separated by the conducting, metal-like, CSs. From the screening theory the overshooting is thought to be a consequence of the decaying IS near the edges of the plateaus; that is, they become evanescent and coexist with ISs from adjoining filling factors which are also in the evanescent regime, leading to current leakage and causing the overshoot effect.

The framework for this model was given a more quantitative explanation of the effect of screening on the edge states when a magnetic field is present by Chklovskii et al. [22] for both integral and fractional states. The main point from this model is that for overshooting to occur the ISs have to enter the evanescent regime [23]. That is, their widths $W_{I S}$ must satisfy the condition $l_{B}<\mathrm{W}_{I S}<\lambda_{F}$. The length scales defining this regime are the magnetic length $l_{B}$ and the Fermi wavelength $\lambda_{F}$. The former is given by $l_{B}=\sqrt{\hbar / e B}$, with $\hbar$ being the reduced Planck's constant, $e$ being the elementary charge, and $B$ being the magnetic field, and the latter is defined as $\lambda_{F}=\sqrt{2 \pi / n_{0}}$, with $n_{0}$ being the bulk electron density of the 2DEG. If $W_{I S}$ is less than $l_{B}$, then the ISs collapse; if $W_{I S}>$ $\lambda_{F}$, then the ISs are well defined. However, within this framework the calculations are performed in a non-self-consistent 
manner. Additionally, assumptions like the $2 \mathrm{DEG}$ being located at the plane of $z=0$ and the electrons being depleted by in-plane metallic gates on the same plane and generally having an oversimplified picture on the boundary conditions lead to an unrealistic model with wide ISs [17,23].

Subsequent work from Lier and Gerhardts [24] and Siddiki et al. [25] combat this by modifying the Chklovskii model with self-consistent calculations and taking into account the Hall bar geometry and chemical etching of the mesa structures, matching, to a great extent, the experimental work in the regime of the IQHE. Examples of experimental work on the IQHE can be found in the works of Sailer et al. [15] and Kendirlik et al. [6], where $\mathrm{Si} / \mathrm{SiGe}$ and GaAs/AlGaAs heterostructures were used, respectively. Some varied results on the temperature behavior of the resistance overshoot effect have been noticed which can be explained by two types of screening models, "the bulk" and "the edge" models [15]. Furthermore, through the theoretical work by Salman et al. [17] the model is extended to include fractional states. Experimentally, the behavior of ISs for fractional states has been studied before, but only in the context of magnetocapacitance [26] and edge magneto-plasmon [27] measurements. Consequently, the aim of this paper is to present this framework for fractional ISs in the context of magnetoresistance measurements, specifically the effect on Hall resistance overshooting. A comparison of the Hall resistance anomalies found not only in the IQH regime but also in the $\mathrm{FQH}$ regime between the theory of self-consistent calculations and experimental data obtained is discussed in Sec. IV.

\section{A. Electron density model}

In order to calculate $W_{I S}$, first, the depletion layer's width $l_{d}$, i.e., the area between the edge of the mesa and the boundary of the 2DEG, a compressible region, has to be calculated. This is given by the empirical formula defined by Salman et al. [23] as

$$
l_{d}=\frac{\pi}{n_{0} a_{B}^{*}}\left(\frac{d_{2 \mathrm{deg}-\mathrm{srf}}}{d_{\text {sample }}}\right)\left[c_{3}-\frac{d_{\mathrm{dnr}-\mathrm{srf}}}{c_{3} a_{B}^{*}} e^{\left(-\frac{d_{e}}{10 a_{B}^{*}}\right)}\right] .
$$

From Eq. (1), $a_{B}^{*}$ is the effective Bohr radius given by $a_{B}^{*}=$ $\frac{\hbar^{2} \epsilon}{m_{e}^{*} e^{2}}$, with an effective mass $m_{e}^{*}=0.067$ calculated using the method described in Ref. [28] and a permittivity for GaAs given by $\epsilon=\epsilon_{r} \epsilon_{0}=12.4 \epsilon_{0}$, with $\epsilon_{0}$ being the permittivity of free space [17]. The constant $c_{3}$ is referenced as $\sim 4.5$ from Salman et al. [23]. In addition $d_{2 \operatorname{deg}-\mathrm{srf}}=100 \mathrm{~nm}$ is the depth of the 2DEG from the surface of the mesa, $d_{\text {sample }}=500 \mu \mathrm{m}$ is the thickness of the wafer, $d_{\mathrm{dnr}-\mathrm{srf}}=60 \mathrm{~nm}$ is the depth at which the donors are located from the surface, and $d_{e}=$ $130 \mathrm{~nm}$ is the depth by which the sample was chemically etched in order to form the mesa for the Hall bar. For this sample the depletion length was estimated as $l_{d} \simeq 20 \mathrm{~nm}$.

Then by using the self-consistent calculations which take into account the geometry of the Hall bar the electron density distribution is estimated by [17]

$$
n_{e l}(x)=n_{0}\left(1-e^{-\left(|x-| W_{H}-l_{d}||\right) / t}\right),
$$

where $x$ is the position along the width, $W_{H}=80 \mu \mathrm{m}$, of the Hall bar and $t \simeq 10 a_{B}^{*}$ is an empirical parameter which specifies the slope of the electron density profile [17]. An

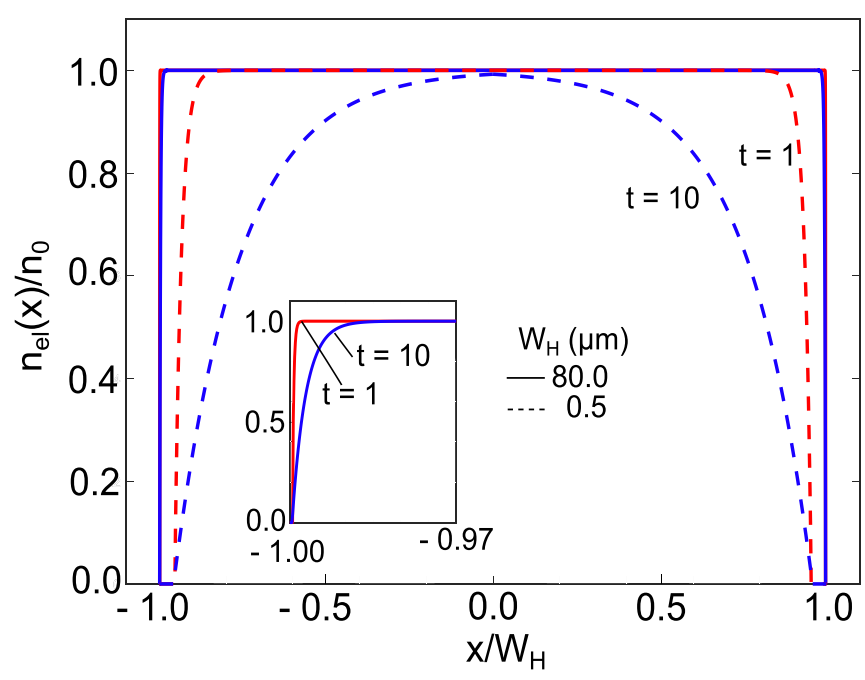

FIG. 2. Density profile of the sample shown as a plot of the density ratio $n_{e l}(x) / n_{0}$ versus $x / W_{H}$. The solid lines are for the Hall bar with $W_{H}=80 \mu \mathrm{m}$, and the dashed ones are for $W_{H}=0.5 \mu \mathrm{m}$. The inset shows a zoomed-in section of the density profile for the $W_{H}=80 \mu \mathrm{m}$ Hall bar. For each $W_{H}$ the effect of a couple of $t$-parameter values are also shown.

example of how the density profile varies with different Hall bar widths and $t$-parameter values is shown in Fig. 2. The local filling factor $v$ at both the integer and fractional ISs is specified by

$$
v\left(x_{k, f}\right)=\pi l_{B}^{2} n_{e l}\left(x_{k, f}\right)=\{k, f\},
$$

where $k=1,2,3, \ldots$, as it represents the integer values, and $f$ takes fractional values corresponding to the respective fractional states [17]. Furthermore, by using Eqs. (2) and (3) the expression for the central position of the ISs can be established as

$$
x_{k, f}=\left|W_{H}-l_{d}\right|+t \ln \left(1-\{k, f\} / v_{0}\right)
$$

if the $\{k, f\}<v_{0}$ condition holds, with $\nu_{0}=\pi l_{B}^{2} n_{0}$ being the bulk filling factor [17].

\section{B. Finite wave functions and integer states' widths}

The analytical expression for calculating the IS widths with integer $v$ is provided by $[17,22]$

$$
a_{k}=\sqrt{\frac{2 \epsilon \Delta E}{\pi^{2} e^{2} d n_{e l}(x) /\left.d x\right|_{x=x_{k}}},}
$$

where $d n_{e l}(x) /\left.d x\right|_{x=x_{k}}$ is the derivative of the density and $\Delta E$ is the energy gap between the adjacent quantized levels. The expression can be modified by taking into consideration the Thomas-Fermi approximation (TFA) and the modified density profile, as explained in Refs. [17,29], to give the equation

$$
a_{k}^{\mathrm{TFA}}=\sqrt{\frac{4 \alpha_{k} a_{B}^{*} t}{\pi\left(v_{0}-k\right)}},
$$

where $\alpha_{k}$ is a dimensionless strength parameter and gives the ratio of $\Delta E$ between consecutive $v$ (different for odd and even $\nu$ ) by taking into account the Zeeman energy $g^{*} \mu_{B} B$ and the 
cyclotron energy $\hbar \omega_{c}$, with $\omega_{c}$ being the angular frequency of the electrons [17,22]. The $\alpha_{k}$ parameter is given as [23]

$$
\alpha_{k}=\frac{\Delta E_{k}}{\hbar \omega_{c}}= \begin{cases}\left(g^{*} \mu_{B} B\right) / \hbar \omega_{c}, & k=\text { odd, } \\ \left(\hbar \omega_{c}-g^{*} \mu_{B} B\right) / \hbar \omega_{c}, & k=\text { even. }\end{cases}
$$

The effective Landé $g^{*}$ factor used in the calculations was estimated as $g^{*}=10.95$ using the method described in Refs. [30,31]. Additionally, $\mu_{B}$ is the Bohr magneton.

By using the TFA to calculate the widths of the ISs, it should be noted that the finite widths of the wave functions are neglected. Therefore, it is feasible for only slowly changing potentials on the magnetic length scale. In contrast to this, for the condition $a_{k}^{\text {TFA }} \lesssim l_{B}$ the TFA is invalid. In order to counterbalance this, Refs. [17,29,32] proposed the use of the quasi-Hartree approximation (QHA), as finite widths of the wave functions can be included through substitution of the $\delta$ function TFA with Landau wave functions. By doing this, however, the energy eigenvalues are still described as in the TFA. The widths of the ISs, within the QHA, can be approximated by $[17,29,32]$

$$
a_{k}^{\mathrm{QHA}}=\left(1-\frac{l_{B}}{a_{k}^{\mathrm{TFA}}}\right) a_{k}^{\mathrm{TFA}} .
$$

By comparing the widths calculated by the two different approximations with the $l_{B}$ scale, three regimes can be identified. The first regime is under the condition that $a_{k}^{\text {TFA }}<l_{B}$ is satisfied and the cyclotron motion of the electron loses its quantization; hence, the system exhibits classical Hall effect characteristics [17]. In contrast under the conditions of $a_{k}^{\mathrm{QHA}}>l_{B}$, the IS with $v=k$ becomes wider than the extent of the wave, resulting in the bulk and the opposing sample edges decoupling, and the IQHE is observed [17]. However, this is valid only as long as $v_{0}<k$. Last but not least, under the circumstances that $a_{k}^{\mathrm{TFA}}<l_{B}<a_{k}^{\mathrm{QHA}}$ the IS enters the evanescent phase [17]. As mentioned earlier, in this situation the electrons are able to tunnel across the strip, with the backscattering being enhanced. Consequently, the Hall resistance displays a deviation from the quantized resistance value, i.e., overshooting. This theoretical concept applies to the fractional states as well, but with slight modifications, which will be discussed in the following section.

\section{Fractional states' widths}

For the fractional states the CF picture by Jain [33] is used for this study. The filling factors $v$ of electrons and CFs are linked by the equation [17]

$$
v=\frac{v^{*}}{2 p v^{*} \mp 1},
$$

where $v^{*}$ stands for the filling factor of the CFs and $p$ is an integer determining the order of the fractional state [17,33]. The energy gap expression for the fractional $v$ is given by [34]

$$
\Delta f=c_{f} \frac{e^{2}}{\epsilon l_{B}},
$$

where $c_{f}$ is a coefficient determined by the corresponding filling factor. The value of $c_{f}$ ranges between 0.06 and 0.11 for the fractional states $1 / 3$ and $2 / 3$ [34]. For simplicity the value for the fractional state calculations performed within this paper is taken as $c_{f}=0.11$ and set as $p=1$. Note that from the literature the difference in size of the energy gaps calculated using the various values of $c_{f}$ within the aforementioned range is negligible in our calculations, so it does not influence the results discussed later [17]. By substituting Eqs. (2) and (10) in Eq. (5), one obtains

$$
a_{f}^{\mathrm{TFA}}=\sqrt{\frac{4 l_{B} c_{f} t}{\pi\left(\nu_{0}-f\right)},}
$$

which provides $W_{I S}$ for fractional states.

Chklovskii et al. [22] proposed that regimes similar to the ones mentioned earlier for the integral states occur by comparing $W_{I S}$ of fractional states with the $l_{B}$ scale. On the other hand, in Ref. [17], it was recommended that the comparison should occur with the cyclotron radius $r_{c f}$ of the CFs instead of $l_{B}$. The $r_{c f}$ for the CFs can be estimated by

$$
r_{c f}=l_{B} \sqrt{2\left(2 p v^{*}+2 p+1\right)} .
$$

This was based on later theories by Chklovskii [35] on the formation of fractional edge states using the CF theory. If this method of comparison is used, then a normalized cyclotron radius $r_{c}=R_{c} / \sqrt{2}=l_{B} \sqrt{\frac{2 n-1}{2}}$, where $n$ is the LL index, for the integer states should be used as well for a more sustainable comparison between integer and fractional states. Hence, identically to the previous section, the evanescent ISs for fractional states exist within the regime of $a_{f}^{\text {TFA }}<r_{c f}<a_{f}^{\text {QHA }}$, where $a_{f}^{\mathrm{QHA}}$ is the equivalent of $a_{k}^{\mathrm{QHA}}$ but for fractional states and calculated using Eq. (8) by substituting $a_{k}^{\text {TFA }}$ with $a_{f}^{\text {TFA }}$ This aspect on which length scale should be used for the comparison will be discussed in more detail in relation to the data presented in a later section.

\section{Current and ISs}

For the regime where the ISs are well established the current flows entirely within them. However, when the ISs break down, the current flows increasingly in the bulk of the system. The Hall resistance is given by the equation

$$
\rho_{x y}^{v}=\frac{V_{\text {Hall }}}{I_{0}}=\frac{h}{e^{2} v},
$$

which is derived in detail in Refs. $[15,17]$, with $I_{0}$ being the current flowing along the Hall bar. From the equation it can be seen that as the IS breaks down, the current density decreases, and thus, the Hall resistance must drop. Nonetheless, when more than one IS is in the evanescent phase, then the current density increases locally, leading to an increase in the Hall resistance as the local filling factor $v(x)$ also increases [17]. Therefore, for the case in which multiple evanescent ISs coexist, the Hall resistance is modified to

$$
\rho_{x y}^{v}=\frac{h}{e^{2}}\left(\frac{1}{v_{1}}\left(I_{1}\right)+\frac{1}{v_{2}}\left(I_{2}\right)\right),
$$

where $v_{1}$ and $v_{2}$ are consecutive filling factors corresponding to an IS with $v_{2}$ having a higher $v$ value, i.e., it is observed at a lower magnetic field than $v_{1}$. Finally, the currents $I_{1}$ and $I_{2}$ correspond to the respective $v$. 
(a)

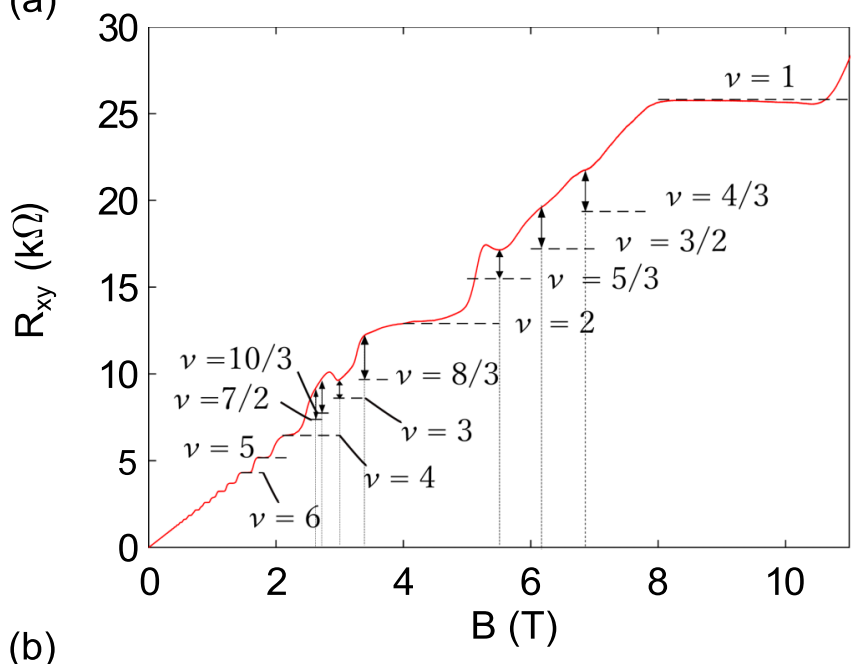

(b)

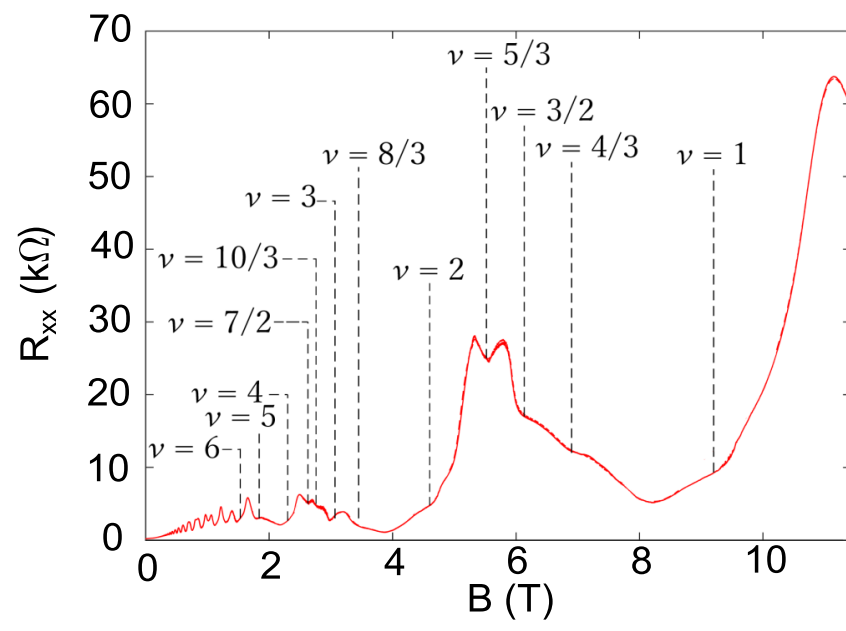

FIG. 3. (a) Plot of $R_{x y}$ as a function of $B$. The horizontal dashed lines indicate the level at which the corresponding $v$, labeled, are supposed to be observed. (b) $R_{x x}$ as a function of $B$. The positions of the corresponding $v$ are indicated by the vertical dashed lines on the curve. The double-sided arrows indicate the location of the magnetic fields and the corresponding overshooting regions.

\section{RESULTS AND DISCUSSION}

The plateaus which correspond to integer and fractional filling factors are observed in the $R_{x y}$ data, as shown in Fig. 3(a). However, overshoot anomalies are noticed for the fractional states $4 / 3,3 / 2,5 / 3,8 / 3,10 / 3$, and $7 / 2$ and the integer $v=3$. As seen from Fig. 3(a), the plateaus for the fractional states $4 / 3,3 / 2,5 / 3,8 / 3$ are well above the dashed lines which correspond to these filling factors. For fractional states at $10 / 3$ and $7 / 2$, although no distinct plateaus are seen in $R_{x y}$, there are striking minima which seem to occur in the $R_{x x}$ measurement corresponding to these fractional states. The reason for the plateaus, corresponding to these fractional states, not being observed is attributed to the fact that there is a large overshooting effect which seems to occur between the $B$ range of 2.8 and $3.1 \mathrm{~T}$, which encapsulates these fractional states as well as the integer $v=3$ state. An important observation is that the $B$ positions of the minima observed in the $R_{x x}$ measurement match the $B$ positions where the maximum width of the ISs is located by using the Eqs. (6) and (8) for the integer ISs and the equivalent ones for the fractional states, as seen in Figs. 3 and 4.

In Fig. 4 the crescents indicate how the ISs for the various $v$ change in width as $B$ increases. The red crescents represent $W_{I S}$ of the odd-integer $v$, the blue ones show the even-integer $v$, and the green ones show the fractional $v$. Note that at the lower $B$ the ISs are much narrower and are the evanescent regions of the ISs. $W_{I S}$ for the integer states decreases for $v$ values at smaller $B$. This is also why the plateaus of the corresponding $v$ decrease in width. Additionally, notice how the ISs in green intercept the various integer $v$ ISs, which is why the overshooting effect is noticed.

Similarly, in Fig. 5, the evolution of the widths of the ISs can be seen for the odd-integer $v$ in red, even integers in blue, and the fractional states in green. The solid lines represent the evolution of $W_{I S}$ as calculated using the TFA, and the dashed lines represent the ones using QHA. The dashed black line is $\lambda_{F}$, the solid black line is $r_{c f}$, and the dash-dotted pink line is $\mathrm{1}_{B}$. By analyzing the data it can be seen that for the regime $l_{B}<a_{k}^{\mathrm{TFA}}, a_{k}^{\mathrm{QHA}}<\lambda_{F}$, i.e., the evanescent regime of the ISs, there are significant overshoot effects occurring. Also notice how for $v=3,10 / 3$, and $7 / 2$, where the overshooting effect is more pronounced, there is a larger overlap of evanescent regions, which explains why the plateaus are more difficult to distinguish in $R_{x y}$ as opposed to other $v$. The relatively large overshoot noticed for $v=3$ is in agreement with measurements stated by Kendirlik et al. [6]. Furthermore, note that our data seem to be in contrast to a suggestion that $r_{c f}$ should be considered the minimum length scale in defining the evanescent regime, as opposed to $l_{B}$. This is illustrated in the example of $v=5 / 3$, where if $r_{c f}$ were considered, it would suggest from our calculations in Fig. 5 that no overshooting should occur as there are no other ISs overlapping the evanescent region of the IS of $v=5 / 3$. However, we can see from Fig. 3(b) that this is clearly not true and is more compatible with the definition of the evanescent regime being defined by $l_{B}$ as this seems to indicate an overlapping of the evanescent ISs of multiple $v$, e.g., $v=1,4 / 3,3 / 2$, and 5/3.

Additionally, by varying the temperature of the sample the evolution of the plateaus was examined (see Fig. 6). The temperature was varied from base temperature (BT) to $2 \mathrm{~K}$. As can be seen in Fig. 6, by increasing the temperature the overshooting is suppressed, with the weak plateaus for $v=4 / 3$ and $3 / 2$ vanishing almost instantly with increasing temperature and finally leading to a steeper change in $R_{x y}$ between the $v=1$ and $v=2$ plateaus. However, the $5 / 3$ fractional state seems to persist for higher temperatures. Initially, it seems that it flattens out at $500 \mathrm{mK}$ and drops in resistance value but still retains an enhanced Hall resistance compared to what is expected theoretically. Around $800 \mathrm{mK}$ it seems to drop down to a value that matches the dashed black line, marking the expected value, and eventually smears out by $2 \mathrm{~K}$. Also note how the right-hand side of the $v=2$ plateau also drops down to match the expected value for this filling factor in the upper inset of Fig. 6.

Similarly, the plateau corresponding to $v=3$ seems to flatten out as the temperature is increased to $2 \mathrm{~K}$ (see the lower inset in Fig. 6). Also, in contrast to the 5/3 state, the enhanced resistance seems to increase with increasing temperature with 


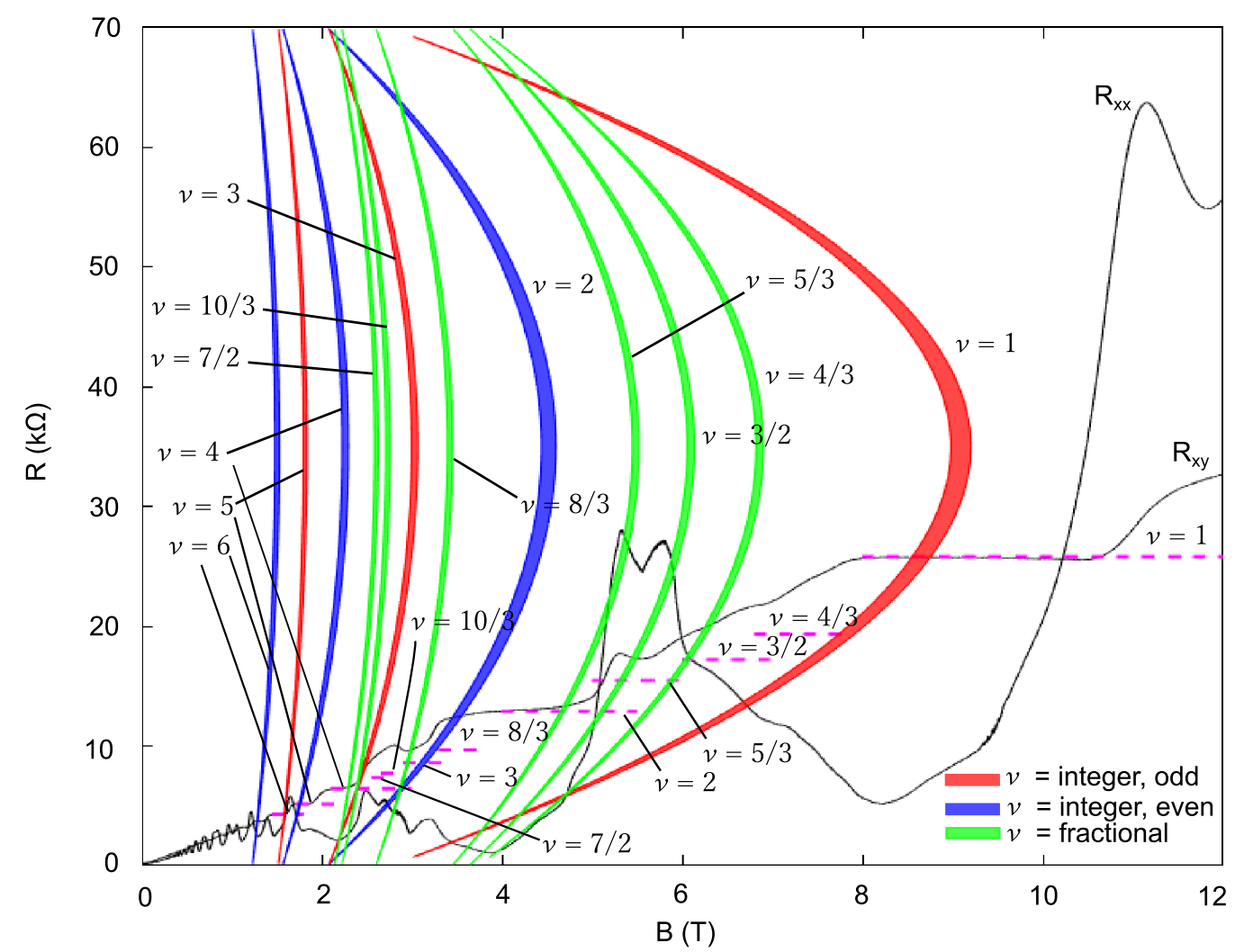

FIG. 4. Plot of $R$ as a function of $B$. The crescents represent the evolution of $W_{I S}$ for the integral and fractional states. The red ones are for odd-integer states, the blue ones are for even integers, and the green ones are for fractional states. The dashed magenta lines correspond to the expected resistance values for each filling factor.

the plateau moving through the $8 / 3$ state and evolving to a flat region at approximately the $7 / 3$ fractional state. This temperature dependence of $v=3$ is in agreement with a previous

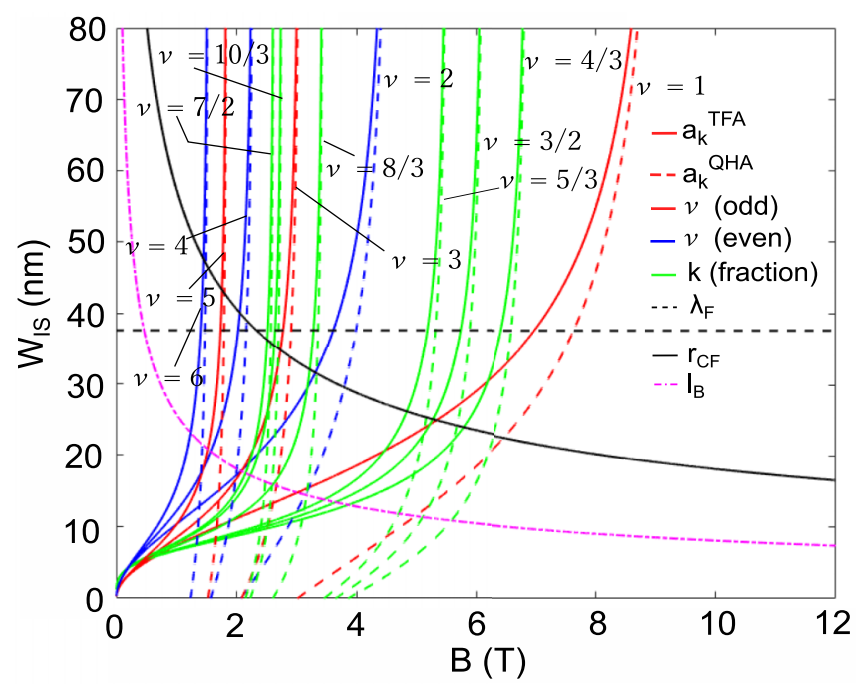

FIG. 5. Plot of $W_{I S}$ versus $B$ by using the TFA (solid red, blue, and green lines) and by using the QHA (dashed red, blue, and green lines). Red is for odd-integer $v$, blue is for even ones, and green is for fractional states. The dashed black line is $\lambda_{F}$. The solid black line is $r_{c f}$, and the dash-dotted pink line is $l_{B}$. study by Kendirlik et al. [6]. This may be attributed to the fact that the IS for $v=3$ is much narrower than the $v=2$ IS, which does match our theoretical calculations (see Fig. 4), with $a_{3}^{\text {TFA }}$ being 2.4 times smaller than $a_{2}^{\text {TFA }}$ and $a_{3}^{\mathrm{QHA}}$ being 3.5 times smaller than $a_{2}^{\mathrm{QHA}}$. It is suggested that as a result at the lower $B$ end of the $v=3$ plateau the bulk strip is narrower than the edge strip of the $v=2$ IS. This, as a result, leads to the overshoot being enhanced as the temperature increases as the $v=3$ IS breaks down at higher temperatures and the $v=2$ IS overpowers it. Moreover, in further compliance with Ref. [6], the overshoot effect is seen only at the low field end of the odd-integer $v$. This is explained as being a consequence of the alternating gap size between the integer states being $\Delta E_{\text {even }} \gg \Delta E_{\text {odd }}[6]$.

Further studies were done by leaving the temperature constant at the BT and varying the current across the Hall bar. Similar to the temperature dependence result, the overshoot effect diminishes as the plateaus flatten out by increasing the current from 10 to $4550 \mathrm{nA}$. However, the overshoot effect corresponding to the fractional states $v=10 / 3$ and $7 / 2$ and the integer state $v=3$ does not increase in resistance value as the current is increased but rather drops down towards the expected resistance value corresponding to $v=3$, i.e., $R_{K} / v$, where $R_{K}$ is the von Klitzing constant equal to $R_{K}=$ $h / e^{2}$. Although this might be in contrast to the temperature measurements, it is once again in agreement with the measurements from Kendirlik et al. [6]. Initially, for the $v=5 / 3$ state the plateau flattens out completely and drops to its $3 R_{K} / 5$ 


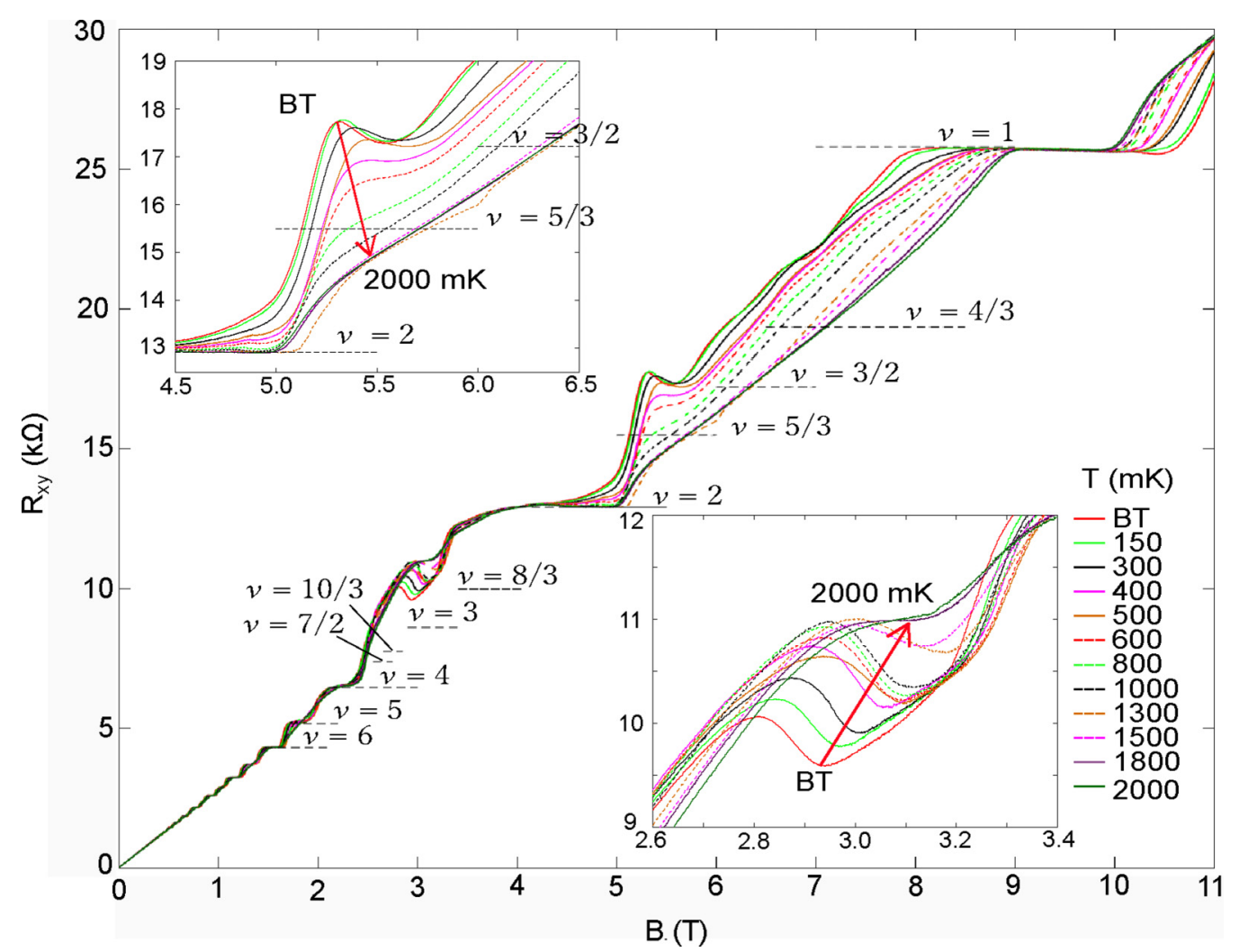

FIG. 6. Plot of $R_{x y}$ versus $B$ at varying temperatures. The upper and lower insets show zoomed-in sections of the graph. The red arrows show the evolution of the $v=5 / 3$ and 3 plateaus, respectively. The dashed black lines are the expected values for $v$, as labeled.

resistance value by a current value of $640 \mathrm{nA}$ but still persists for higher current values, but its resistance value keeps dropping. In addition the $4 / 3$ fractional state plateau persists up to a current of $100 \mathrm{nA}$. This can be seen in Fig. 7, where the dashed black lines indicate the evolution for some of the filling factor plateaus which exhibit overshooting behavior. For the $v=3$ state the maximum current, i.e., $4550 \mathrm{nA}$, had to be applied in order for the plateau to become completely flat. However, it should be noted that above the applied current of

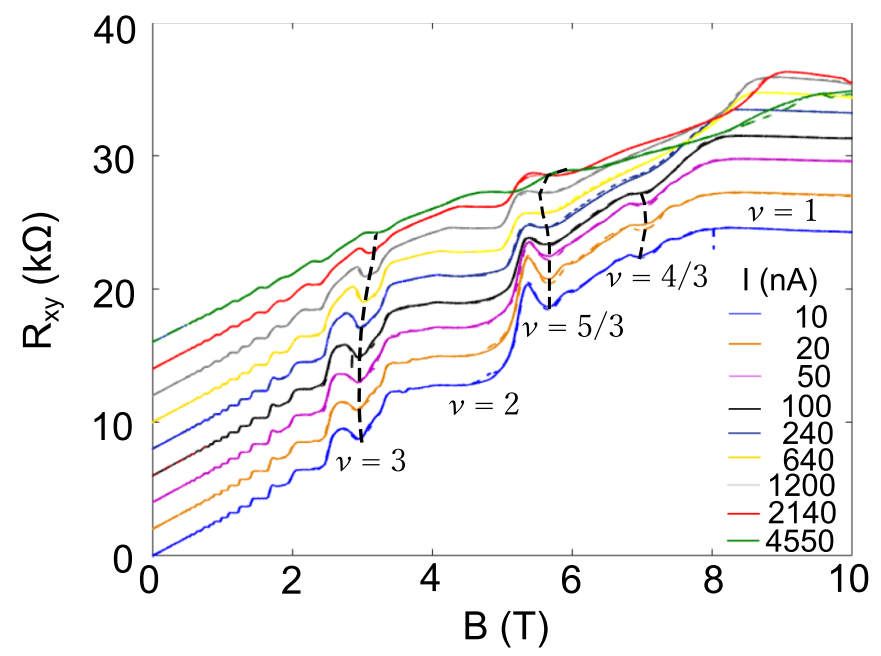

FIG. 7. Plot of $R_{x y}$ versus $B$ for different sample currents. The dashed black lines show the evolution of the stated $v$.
$640 \mathrm{nA}$, although the plateaus are flatter, their corresponding resistance falls below the defined $R_{K} / v$ value for the plateaus corresponding to filling factors values $v<3$. This could be due to the higher currents breaking down the IS.

One could suggest that this change in the behavior of the plateaus by increasing the current is due to an increase in the bulk current density $j=I / W_{H}$ [15]. However, this is in contrast to the follow-up data presented in Fig. 8. Here the current was fixed at $10 \mathrm{nA}$, and the temperature was held

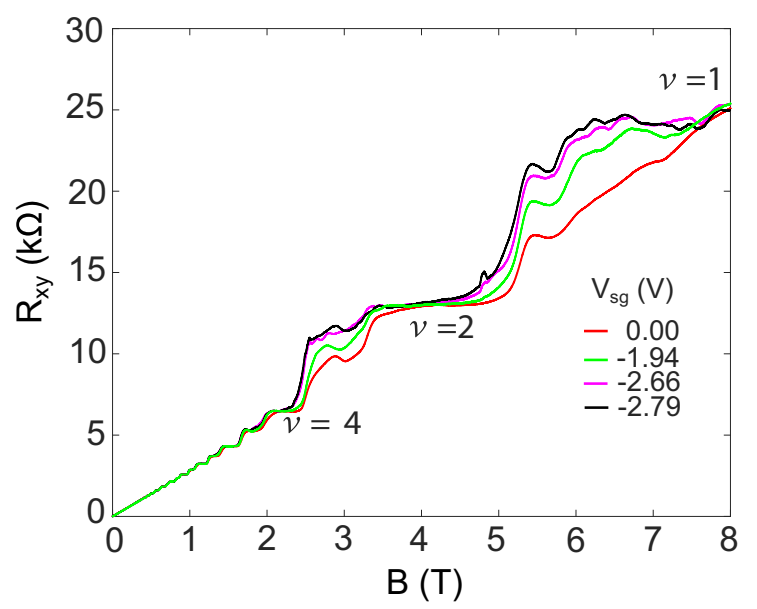

FIG. 8. Plot of $R_{x y}$ versus $B$ for different $V_{s g}$ applied on the split gates. 
at BT, but the size of the channel was altered by depleting the electrons under the application of $V_{s g}$, resulting in an increase in current density. From Fig. 8 it can be seen that by increasing $V_{s g}$ the overshooting is enhanced for all the integer and fractional states mentioned earlier and even seems to create an overshooting effect for the $v=5$ state. Therefore, thinking that the average bulk current density $j$ has an effect on the overshoot effect would be rather naive. Consequently, the current $I$ is the only decisive parameter on the evolution of the overshooting. This is compatible with the work by Sailer et al. [15], with the only difference being that the constrictions were achieved lithographically as opposed to the electrostatic methods in our case. Rather, the fact that the overshoot effect increases by decreasing the channel size indicates that the anomalous increase in the Hall resistance is not related to the bulk of the 2DEG [15].

The data fit well with the idea of the screening theory; that is, as the electrons leak out of an IS within the evanescent regime, the electron gas will be heated up locally. The electrons will then scatter in the nearby compressible region at the low- $B$ end of a Hall plateau. The first IS to be affected will be the outer one as the overshoot is destroyed with increasing sample current. Nevertheless, the inner IS is less affected, leading to a preserved Hall plateau. Second, another consequence of the increased currents through the sample is the tilting of the potential landscape [36] in the outof-linear-response regime. Both evanescent ISs at one edge boundary become wider at the expense of both ISs at the edge boundary on the other side. For a certain current amplitude, the narrowest outer IS breaks down, resulting in a breakdown of the overshoot [15].

The channel's size dependence on the overshoot can be explained as a consequence of the current starting to leak out of the inner IS at the lower magnetic end of the plateaus, and it redistributes to the adjacent resistance minimum [15]. As the channel is further constricted, less current flows in the bulk. Therefore, more current is confined within the adjacent IS which is in an evanescent state and is at a local resistance minimum. The result is that the overshoot amplitude increases due to the redistribution of the current between the evanescent IS and the bulk [15].

Also by using Eq. (14) and comparing the areas overlapping within the evanescent regime the amount of overshooting can be estimated to a good approximation. For example, for $v=5 / 3$ the overshoot is measured as $\sim 17390 \mathrm{k} \Omega$. By calculating the areas of the overlapping currents for $v=5 / 3$, that is, taking into account the overlapped areas of $v=1, v=4 / 3$, $v=3 / 2$, and $v=5 / 3$, from Fig. 5 and assuming the sum of the areas enclosed for each IS is proportional to the total current in the system, one can estimate the overshooting for $v=5 / 3$ as $\sim 17600 \mathrm{k} \Omega$. The discrepancy is small enough to be considered a good match between the two values.

Further studies could lead to a better understanding of harnessing and manipulating the powers of the QHE for future technologies, like quantum computers and metrology. The incompressible nature of the edge states is an ideal candidate to implement semiconductor logic gates for flying-qubit quantum computing architectures due to their large coherence length [37]. Additionally, fractional states, e.g., the 5/2 state, are thought to lead to topologically protected non-Abelian states used for quantum computing [38], and the Landau quantization was shown to be of importance in storing energy due to high magnetocapacitance at both integer and fractional states due to the energy gap forming [39].

\section{CONCLUSIONS}

We have shown the presence of an anomalous increase in the Hall resistance (overshoot) for both integral and fractional states. The self-consistent screening theory calculations for the incompressible and compressible states for both these types of states are in good agreement with the data presented in this paper. The calculations took into account both the dimensions of the Hall bar and the fact that the device was etched. The various observations with the evolution of the overshooting by changing the parameters of temperature, sample current, and channel constriction via electrostatic methods are all well understood with regards to the screening theory.

\section{ACKNOWLEDGMENTS}

This work was funded by the Engineering and Physical Sciences Research Council (EPSRC), United Kingdom (EP/R029075/1) and the United Kingdom Research and Innovation (UKRI) Future Leaders Fellowship (MR/S015728/1).
[1] K. Klitzing, G. Dorda, and M. Pepper, New Method for HighAccuracy Determination of the Fine-Structure Constant Based on Quantized Hall Resistance, Phys. Rev. Lett. 45, 494 (1980).

[2] D. C. Tsui, H. L. Stormer, and A. C. Gossard, Two-Dimensional Magnetotransport in the Extreme Quantum Limit, Phys. Rev. Lett. 48, 1559 (1982).

[3] H. Fu, P. Wang, P. Shan, L. Xiong, L. N. Pfeiffer, K. West, M. A. Kastner, and X. Lin, Competing $v=5 / 2$ fractional quantum Hall states in confined geometry, Proc. Natl. Acad. Sci. USA 113, 12386 (2016)

[4] S. Ilani, J. Martin, E. Teitelbaum, J. H. Smet, D. Mahalu, V. Umansky, and A. Yacoby, The microscopic nature of local- ization in the quantum Hall effect, Nature (London) 427, 328 (2004).

[5] E. Ahlswede, P. Weitz, J. Weis, K. von Klitzing, and K. Eberl, Hall potential profiles in the quantum Hall regime measured by a scanning force microscope, Phys. B (Amsterdam, Neth.) 298, 562 (2001)

[6] E. M. Kendirlik, S. Sirt, S. B. Kalkan, W. Dietsche, W. Wegscheider, S. Ludwig, and A. Siddiki, Anomalous resistance overshoot in the integer quantum Hall effect, Sci. Rep. 3, 3133 (2013).

[7] S. Kumar, K. J. Thomas, L. W. Smith, M. Pepper, G. L. Creeth, I. Farrer, D. Ritchie, G. Jones, and J. Griffiths, Many-body 
effects in a quasi-one-dimensional electron gas, Phys. Rev. B 90, 201304 (2014).

[8] S. Kumar, M. Pepper, S. N. Holmes, H. Montagu, Y. Gul, D. A. Ritchie, and I. Farrer, Zero-Magnetic Field Fractional Quantum States, Phys. Rev. Lett. 122, 086803 (2019).

[9] M. Büttiker, Four-Terminal Phase-Coherent Conductance, Phys. Rev. Lett. 57, 1761 (1986).

[10] A. Alphenaar, P. L. McEuen, R. G. Wheeler, and R. N. Sacks, Selective Equilibration Among the Current-Carrying States in the Quantum Hall Regime, Phys. Rev. Lett. 64, 677 (1990).

[11] P. L. McEuen, A. Szafer, C. A. Richter, B. W. Alphenaar, J. K. Jain, A. D. Stone, R. G. Wheeler, and R. N. Sacks, New Resistivity for High-Mobility Quantum Hall Conductors, Phys. Rev. Lett. 64, 2062 (1990).

[12] H. Zheng, K. Choi, D. Tsui, and G. Weimann, Size effect in the quantum Hall regime, Surf. Sci. 170, 209 (1986).

[13] C. Richter, R. Wheeler, and R. Sacks, Overshoot of quantum Hall plateaus, Surf. Sci. 263, 270 (1992).

[14] S. Komiyama and H. Nii, Nonequilibrium electron distribution and nonlocal resistance in a two-dimensional electron gas at high magnetic fields, Phys. B (Amsterdam, Neth.) 184, 7 (1993).

[15] J. Sailer, A. Wild, V. Lang, A. Siddiki, and D. Bougeard, Quantum Hall resistance overshoot in two-dimensional (2D) electron gases: Theory and experiment, New J. Phys. 12, 113033 (2010).

[16] E. Ahlswede, J. Weis, K. V. Klitzing, and K. Eberl, Hall potential distribution in the quantum Hall regime in the vicinity of a potential probe contact, Physica E 12, 165 (2002).

[17] A. Salman, A. I. Mese, M. B. Yucel, and A. Siddiki, Semianalytical model of Hall resistance anomalies (overshooting) in the fractional quantized Hall effect, Eur. Phys. J. B 86, 203 (2013).

[18] J. J. Marě, A. Siddiki, D. Kindl, P. Hubík, J. Krištofik, and K. Krištofik, Electrostatic screening and experimental evidence of a topological phase transition in a bulk quantum Hall liquid, New J. Phys. 11, 083028 (2009).

[19] A. Siddiki, J. Horas, D. Kupidura, W. Wegscheider, and S. Ludwig, Asymmetric nonlinear response of the quantized Hall effect, New J. Phys. 12, 113011 (2010).

[20] C. W. J. Beenakker, Edge Channels for the Fractional Quantum Hall Effect, Phys. Rev. Lett. 64, 216 (1990).

[21] A. M. Chang, A unified transport theory for the integral and fractional quantum Hall effect: Phase boundaries, edge currents, and transmission/reflection probabilities, Solid State Commun. 74, 871 (1990).

[22] D. B. Chklovskii, B. I. Shklovskii, and L. I. Glazman, Electrostatics of edge channels, Phys. Rev. B 46, 4026 (1992).

[23] A. Salman, M. B. Yucel, and A. Siddiki, Edge electrostatics revisited, Phys. E (Amsterdam, Neth.) 47, 229 (2013).
[24] K. Lier and R. R. Gerhardts, Self-consistent calculations of edge channels in laterally confined two-dimensional electron systems, Phys. Rev. B 50, 7757 (1994).

[25] A. Siddiki, S. Erden Gulebaglan, N. Boz Yurdasan, G. Bilgec, A. Yildiz, and I. Sokmen, Evanescent incompressible strips as origin of the observed Hall resistance overshoot, Europhys. Lett. 92, 67010 (2010).

[26] S. Takaoka, K. Oto, S. Uno, K. Murase, F. Nihey, and K. Nakamura, Edge States in the Fractional Quantum Hall Effect Regime Investigated by Magnetocapacitance, Phys. Rev. Lett. 81, 4700 (1998).

[27] G. Ernst, N. B. Zhitenev, R. J. Haug, and K. Von Klitzing, Dynamic Excitations of Fractional Quantum Hall Edge Channels, Phys. Rev. Lett. 79, 3748 (1997).

[28] A. F. Braña, C. Diaz-Paniagua, F. Batallan, J. A. Garrido, E. Muñoz, and F. Omnes, Scattering times in AlGaN/GaN two-dimensional electron gas from magnetoresistance measurements, J. Appl. Phys. 88, 932 (2000).

[29] A. Siddiki, Electrostatic theory of irregular conductance oscillations through a quantum constriction under quantized Hall conditions, Physica E 122, 114145 (2020).

[30] A. Usher, R. J. Nicholas, J. J. Harris, and C. T. Foxon, Observation of magnetic excitons and spin waves in activation studies of a two-dimensional electron gas, Phys. Rev. B 41, 1129 (1990).

[31] T.-Y. Huang, C.-T. Liang, Y. F. Chen, M. Y. Simmons, G.-H. Kim, and D. A. Ritchie, Direct measurement of the spin gaps in a gated GaAs two-dimensional electron gas, Nanoscale Res. Lett. 8, 138 (2013).

[32] A. Siddiki and R. R. Gerhardts, Incompressible strips in dissipative Hall bars as origin of quantized Hall plateaus, Phys. Rev. B 70, 195335 (2004).

[33] J. K. Jain, Composite-Fermion Approach for the Fractional Quantum Hall Effect, Phys. Rev. Lett. 63, 199 (1989).

[34] A. Chang, The Quantum Hall Effect, edited by R. Prange and S. Girvin (Springer, New York, 1989).

[35] D. B. Chklovskii, Structure of fractional edge states: A composite-fermion approach, Phys. Rev. B 51, 9895 (1995).

[36] A. Siddiki, Current-direction-induced rectification effect on (integer) quantized Hall plateaus, Europhys. Lett. 87, 17008 (2009).

[37] P. Bordone, L. Bellentani, and A. Bertoni, Quantum computing with quantum-Hall edge state interferometry, Semicond. Sci. Technol. 34, 103001 (2019).

[38] C. Nayak, S. H. Simon, A. Stern, M. Freedman, and S. Das Sarma, Non-Abelian anyons and topological quantum computation, Rev. Mod. Phys. 80, 1083 (2008).

[39] E. M. Kendirlik, S. Sirt, S. B. Kalkan, N. Ofek, V. Umansky, and A. Siddiki, The local nature of incompressibility of quantum Hall effect, Nat. Commun. 8, 14082 (2017). 